\title{
Cálculo estimativo: un estudio con alumnos de 5to año de primaria
}

\section{Computational estimation: A study with 5th grade primary students}

\author{
Sandra Stauffer ${ }^{1}$ \\ Diana Solares ${ }^{2}$ \\ Claudia Broitman ${ }^{3}$
}

\begin{abstract}
Resumen: En este artículo presentamos resultados de un estudio sobre la enseñanza del cálculo estimativo para la multiplicación y la división. Los análisis preliminares permitieron inventariar y analizar la variedad de problemas en diferentes fuentes y a partir de variaciones de los mismos diseñamos una secuencia de 14 clases para 5to año de la escuela primaria. El estudio se centró en los conocimientos puestos en juego por los alumnos, en las intervenciones didácticas y en las interacciones en las clases. En este trabajo se documentan y analizan en particular las dificultades a las que se enfrentaron los alumnos y los procedimientos usados, tanto correctos como incorrectos, cuando resuelven problemas que implican anticipar si un cociente será mayor, menor o igual al cociente de otro cálculo dado. Entre los marcos teóricos de referencia para el diseño y el análisis de las clases se destacan la Teoría de las Situaciones Didácticas (Brousseau, 2007) y la metodología de la Ingeniería Didáctica (Artigue, 1998). La intención de este estudio es aportar conocimientos sobre la enseñanza del cálculo estimativo al sistema educativo y al desarrollo de futuras investigaciones.
\end{abstract}

Fecha de recepción: 6 de julio de 2018. Fecha de aceptación: 23 de octubre de 2019.

1 Universidad Autónoma de Querétaro. México, stauffersandra@yahoo.com, orcid.org/0000-0001-6688-2139

2 Universidad Autónoma de Querétaro. México, violetasolares@gmail.com, orcid.org/0000-0001-6034-6693

3 Universidad Nacional de la Plata. Argentina, claubroi@gmail.com. orcid.org/0000-0002-1774-6752 
Palabras claves: Cálculo estimativo, problemas multiplicativos, secuencia didáctica, Teoría de las Situaciones Didácticas, escuela primaria.

\begin{abstract}
In this article, we present the results of a study regarding the teaching of estimative calculation for multiplication and division. The preliminary analysis enabled the enumeration and analysis of the variety of the problems in different sources. Using their variations as a starting point, we designed a sequence of 14 lessons for the 5 th grade of primary school. The study focused on the knowledge of the students, the didactic interventions and the interaction during class. This work documents and analyzes the difficulties faced by the students and the procedures used - correct or incorrect- when they solve problems that involve the anticipation of whether a quotient will be higher, lower or equal to that one of another calculation given. The theoretical framework to design and analyze the sequence was the Theory of Didactic Situations (Brousseau, 2007) and the methodology of Didactic Engineering (Artigue, 1998) The aim of this work is not only contribute to the teaching of estimative calculation but also the developing of new didactic investigations.
\end{abstract}

Keywords: Computational estimation, multiplicative problems, didactic sequence, Theory of Didactical Situations, primary school.

\title{
1. INTRODUCCIÓN
}

Presentaremos algunos resultados de un estudio que se propuso indagar la enseñanza del cálculo estimativo en alumnos de tercer ciclo ${ }^{4}$ de la escuela primaria. En particular, analizaremos las dificultades a las que se enfrentan alumnos de quinto grado de primaria al resolver problemas que implican comparar expresiones multiplicativas y los procedimientos usados -tanto correctos como incorrectos-. Los conocimientos de los alumnos se identificaron a lo largo de una secuencia didáctica de 14 clases, diseñada especialmente para este estudio y en el marco de una tesis de maestría (Stauffer, 2018). ${ }^{2}$

El cálculo mental estimativo o aproximado es un tipo de problemas dentro del cálculo mental. A diferencia del cálculo mental exacto, el cálculo estimativo

\footnotetext{
${ }^{4}$ El tercer ciclo en México corresponde a $5^{\circ}$ y $6^{\circ}$ año de primaria.
} 
busca un resultado cuyo grado de aproximación puede variar según el problema. Segovia y Castro (1989) definen la estimación como "juicio sobre el valor del resultado de una operación numérica o de la medida de una cantidad, en función de circunstancias individuales del que lo emite" (p.18). Este tipo de cálculo está presente en la vida cotidiana de nuestra cultura, ya sea para obtener un resultado numérico, como lo señala la cita anterior o, para tomar una decisión. Por ejemplo, los adultos utilizamos principalmente el cálculo estimativo en situaciones de compra-venta donde debe decidirse qué opción conviene más ("¿Un paquete de 18 pastelillos por \$540 es más económico que tres de 6 pastelillos por \$192?"). Ya solo el uso social justificaría su enseñanza en las escuelas primarias; pero además, este tipo de cálculo constituye un importante recurso de control, dado que permite anticipar el rango posible de un resultado y luego revisar la validez del mismo a través de un cálculo exacto.

El cálculo mental estimativo juega un rol importante en el aprendizaje de los conocimientos matemáticos. Investigaciones realizadas con niños por Lerner (2005) y retomadas para estudiantes adultos por Broitman (2012), muestran que el trabajo con ciertos problemas de cálculo mental estimativo contribuye a la construcción de algunas relaciones y características de nuestro sistema de numeración. Se proponen, por ejemplo, problemas en los que los estudiantes tengan que anticipar con qué número empieza el resultado de un cálculo como 56 + 22, u otros donde tengan que decidir si un cálculo como $29+49$ puede dar ochenta y algo o no. Resolviendo este tipo de problemas los estudiantes se enfrentan a tomar decisiones relacionadas con la agrupación decimal y el valor posicional, pilares importantes de nuestro sistema de numeración.

Algunos autores señalan, además, que ciertos problemas de cálculo mental estimativo tienen el potencial de promover procedimientos de tipo algebraico en los alumnos. Mason, Graham, Pimm y Gowar (1985) identifican cuatro raíces del álgebra: aritmética generalizada; posibilidades y restricciones; reordenamiento y manipulación y expresión de la generalidad. Mencionan que la esencia de la raíz de la llamada expresión de la generalidad está en "identificar y aprovechar aquellos momentos cuando los alumnos están realizando cálculos que pueden revelar, con un poquito de indagación, las reglas de la aritmética" (p. 90) y entre otros, Ios cálculos estimativos posibilitarían dicha cuestión. Por ejemplo, decidir si 12 × 12'600 es menor, mayor o igual a 24 × 6300, o la misma situación de los pastelillos anteriormente citada, son problemas que implican un tipo de cálculo que conduce a una práctica algebraica, en lugar de buscar resultados numéricos como suele hacerse en aritmética. 
Por algunas de estas razones u otras, el currículo de México como el de Argentina ${ }^{5}$ incorporan el cálculo estimativo o el cálculo aproximado como contenido en la escuela primaria.

En México, en los "Propósitos del estudio de las Matemáticas para la Educación Primaria" se menciona de manera explícita el cálculo estimativo:

En esta fase de su educación, como resultado del estudio de las Matemáticas se espera que los alumnos utilicen el cálculo mental, la estimación de resultados o las operaciones escritas con números naturales, así como la suma y resta con números fraccionarios y decimales para resolver problemas aditivos y multiplicativos. (Secretaría de Educación Pública, 2011, p. 62)

Sin embargo, los materiales curriculares de apoyo para el maestro contienen pocas propuestas explícitas sobre cómo abordar las también escasas actividades de cálculo estimativo que presentan los libros de texto oficiales. ${ }^{6}$ Por ejemplo, el Libro del maestro de Matemáticas quinto grado (SEP, 2016) -grado en el que se realizó la investigación que aquí se reporta- presenta sugerencias didácticas únicamente para dos lecciones explícitamente dedicadas al cálculo mental (suma y resta de fracciones y de números decimales), y la estimación únicamente se aborda en una lección sobre la estimación del peso.

En Argentina encontramos una mayor presencia del cálculo estimativo en los documentos curriculares, y se reconoce la importancia de que los alumnos lo desarrollen a lo largo de la educación primaria. En los Núcleos de los Aprendizajes Prioritarios (NAP) elaborados por el Ministerio de Educación, Ciencia y Tecnología (2005) para el segundo ciclo de la escuela primaria, se plantea la necesidad de ofrecer situaciones de enseñanza que promuevan "El análisis y el uso reflexivo de distintos procedimientos para estimar y calcular en forma exacta y aproximada" (p. 17). La importancia asignada al cálculo estimativo en este documento de carácter nacional aparece en todos los grados de la escuela primaria.

También numerosos libros de texto de este país presentan propuestas de enseñanza del cálculo estimativo, por ejemplo, Saiz y Parra (2013) y Broitman,

5 Hemos analizado en particular su enseñanza en estos dos países por ser los de nuestros ámbitos de trabajo e investigación.

6 En las escuelas primarias de México se distribuyen gratuitamente libros de texto de Matemáticas y de otras asignaturas. Esos libros son elaborados en la Secretaría de Educación Pública. 
C., Itzcovich, H., Novembre, A., Escobar, M., Grimaldi, V., Ponce, H., y Sancha, I. (2015). Asimismo, ponemos en relieve la existencia de varios materiales producidos para docentes con orientaciones para la enseñanza del cálculo estimativo, por ejemplo, Broitman, C. (2011), y Cálculo mental con números naturales publicado por la Secretaría de Educación del Gobierno de la Ciudad de Buenos Aires (2006).

\section{REFERENCIAS CONCEPTUALES Y DECISIONES METODOLÓGICAS}

La investigación realizada, de la que aquí solo se reporta un aspecto, tuvo la intención de estudiar la enseñanza y el aprendizaje escolar del cálculo estimativo, poniendo énfasis en los tipos de problemas, en las intervenciones didácticas y en los conocimientos de los alumnos, considerando las interacciones entre unos y otros aspectos. Para este fin elaboramos una secuencia didáctica de 14 clases durante la cual trabajamos con tres tipos de problemas diferentes que se presentarán más adelante.

Para diseñar y anticipar la gestión de la clase en torno a los problemas seleccionados, nos hemos apoyado en algunos conceptos centrales de la Teoría de las Situaciones Didácticas de Brousseau (Brousseau, 2007). En particular hemos tomado su noción de devolución, en el sentido del otorgamiento a los estudiantes de la responsabilidad matemática de resolver los problemas propuestos a partir de sus propios puntos de partida. Así, hemos buscado instalar condiciones para que produzcan y transformen sus conocimientos. Otra idea de referencia de esta teoría, y que hemos adoptado para estas clases, es que los alumnos puedan hacerse cargo de la validación de sus decisiones e ideas matemáticas, en lugar de que estas sean solo una responsabilidad del docente cuando determina si las respuestas dadas son o no pertinentes y correctas. Por otra parte, nos resulta también un aporte la concepción brousseauniana de producción colectiva del conocimiento matemático a través de procesos de debate entre los alumnos y conducidos por el docente. En las clases de la secuencia fomentamos estos espacios y documentamos en este trabajo algunos episodios que dan cuenta de ello. Otra noción también central para el diseño de la presente secuencia didáctica es el concepto de institucionalización, el cual hace referencia a las diferentes intervenciones del docente dirigidas a la toma de conciencia de los alumnos de aquello que han producido, poniendo en relación sus conocimientos con determinados saberes matemáticos. El concepto de 
institucionalización se hace presente en las maneras en las que el docente va dirimiendo los avances en los conocimientos producidos por los alumnos.

Con respecto a los conocimientos de los alumnos, se buscaba identificar procedimientos correctos y erróneos, así como las dificultades que podrían aparecer en alumnos de quinto grado de una escuela primaria al resolver problemas de cada tipo de los seleccionados.

Implementamos la secuencia durante los meses de noviembre y diciembre de 2016 en un grupo de $5^{\circ}$ grado de un colegio bilingüe (español-alemán) de la ciudad de Querétaro, México. Aunque la lengua materna de la gran mayoría de los alumnos del colegio es el español, las clases de matemáticas se imparten en alemán. Por lo mismo, decidimos aplicar la secuencia en esa lengua, para no alterar la dinámica establecida en el colegio y reconociendo, además, que la mayoría de los alumnos ya tienen una larga experiencia con ese idioma. Sin embargo, durante la implementación de la secuencia se aceptaron respuestas de los alumnos también en español y, se les invitó a usar su lengua materna en caso de que tuvieran dificultades para expresarse en alemán.

Si bien la elaboración de la secuencia didáctica no fue en sentido estricto una Ingeniería Didáctica (Artigue, 1998), tomamos de dicha metodología los siguientes aspectos: la realización de análisis preliminares incluyendo antecedentes de otros estudios, producciones curriculares, libros de texto y materiales para docentes; el diseño y análisis a priori de las situaciones de enseñanza para anticipar errores, procedimientos, intervenciones didácticas, entre otras cuestiones; una fase de implementación o experimentación y luego, un análisis a posteriori, con su consecuente validación a partir de contrastar anticipaciones y supuestos con los resultados efectivamente obtenidos en la enseñanza.

Los análisis preliminares nos permitieron inventariar los tipos de problemas de cálculo estimativo presentes en diferentes fuentes: investigaciones, producción curricular, libros de texto, materiales para docentes, etc. A partir de un análisis riguroso de esos problemas decidimos seleccionar algunos para nuestro estudio, diseñando una secuencia didáctica en la que se adaptaban o recreaban varios de los tipos de problemas identificados.

Finalmente, la secuencia a implementar abarcaría 14 clases de 45 minutos cada una, durante las cuales se implementaron diferentes tipos de problemas para la multiplicación y para la división, y en cada uno de ellos se comandaban variables didácticas que permitirían graduar la complejidad de los mismos. Asimismo, la secuencia incluía momentos de repaso, de evaluación, de análisis de la evaluación, entre otros. 
Los tipos de problemas que estaban incluidos en la secuencia definitiva fueron: ${ }^{7}$

A. Anticipar si el producto/cociente es menor, igual o mayor a un número dado Ejemplo:

$260 \div 24$ da

O menos que 10

$O$ igual a 10

O más que 10

B. Encuadrar el producto/cociente entre números dados

Ejemplo:

$5940 \div 24 \mathrm{da}$

O entre 0 y 10

○ entre 10 y 100

O entre 100 y 1000

C. Anticipar si el producto/cociente será mayor, menor o igual al resultado de otro cálculo dado

Ejemplo:

Decide si el cociente de $24^{\prime} 800 \div 6$ es mayor, menor o igual al cociente de $12^{\prime} 400 \div 3{ }^{8}$

Cada uno de estos tres tipos de problemas se plantearon para la multiplicación y luego para la división. Además, dentro de cada tipo se secuenciaban por grado de dificultad variando los números y las relaciones entre los números.

Al terminar la implementación de la secuencia hicimos un análisis exhaustivo de las intervenciones didácticas y de los procedimientos, errores y dificultades de los alumnos al resolver cada tipo de problemas. Presentaremos únicamente los errores y dificultades que manifestaron los alumnos al resolver problemas del tipo descrito en el inciso C y solamente para la división: anticipar si el cociente será mayor, menor o igual al cociente de otro cálculo dado.

\footnotetext{
7 Por razones de espacio no se explicita en este artículo el análisis a priori de la tipología de problemas que permitió construir criterios de elección y secuenciación de tipos de problemas.

${ }^{8}$ Si bien la norma es el Sistema Internacional de Medidas (SI) que propone separar los números en grupos de tres cifras con un espacio, hemos decidido preservar en este artículo la escritura utilizada con los alumnos, forma generalizada en la escuela donde se implementó la secuencia.
} 


\section{ERRORES Y DIFICULTADES DE LOS ALUMNOS EN PROBLEMAS QUE IMPLICAN ANTICIPAR SI EL COCIENTE SERÁ MAYOR, MENOR O IGUAL AL COCIENTE DE OTRO CÁLCULO DADO}

Dentro de la secuencia didáctica mencionada se propusieron a los alumnos problemas que exigían anticipar si el cociente de un cálculo sería mayor, menor o igual al cociente de otro cálculo dado. En el interior de este tipo de problemas se pueden distinguir:

- aquellos donde solamente se variaba el dividendo o el divisor (p.ej. comparar $24^{\prime} 800 \div 6$ con $24^{\prime} 800 \div 12$, o comparar $24^{\prime} 800 \div 6$ con $12^{\prime} 400 \div 6$ )

- aquellos donde se variaban simultáneamente el dividendo y el divisor (p.ej. comparar $24^{\prime} 800 \div 6$ con $12^{\prime} 400 \div 3$ ).

Los problemas donde solamente era diferente el dividendo o el divisor causaron poca dificultad. En cambio, los problemas donde el dividendo y el divisor eran diferentes sí dieron lugar a variados errores. Respecto a estos últimos problemas trabajamos con diferentes variantes:

- se multiplican/dividen el dividendo y el divisor por/entre el mismo número (Ejemplo: comparar 24'800 $\div 6$ con 12'400 $\div 3$; el cociente sigue igual);

- se multiplica uno de los dos por un número, mientras que el otro se divide entre el mismo número (Ejemplo: comparar $24^{\prime} 800 \div 6$ con $12^{\prime} 400 \div 12$; el cociente aumenta/disminuye)

- se agregan/quitan mil al dividendo mientras que al divisor se le quita/ agrega uno (Ejemplo: comparar $24^{\prime} 800 \div 6$ con $23^{\prime} 800 \div 7$ ).

En una evaluación que hicimos a los alumnos en la clase número 10 de la secuencia didáctica, identificamos que en las dos primeras variantes hubo errores similares a los que se habían manifestado anteriormente en la comparación de multiplicaciones: aparentemente más de la mitad de los alumnos se confundió en cuanto a las relaciones que se pueden establecer entre dos multiplicaciones y las que se pueden establecer entre dos divisiones. En la comparación de dos multiplicaciones (p. ej. 12 x 12'600 con 24 x 6300) se puede establecer que si se multiplica uno de los factores por un número y se divide el otro entre el mismo número, se mantiene el resultado. Sin embargo, esta relación no es válida al comparar dos divisiones. En el problema de decidir 
si $24^{\prime} 800 \div 3$ da lo mismo que $12^{\prime} 400 \div 6$, doce de veinte alumnos dijeron, erróneamente, que sí da lo mismo; todos ellos explicaron su respuesta diciendo que se multiplicó el dividendo por 2 y se dividió el divisor entre 2, y que por eso "se compensa". Veamos la explicación de José: "Da lo mismo porque el dividendo es lo doble y el divisor es la mitad, y da lo mismo porque el dividendo se hace lo doble y el divisor se hace la mitad."

Algo parecido surgió en el problema de comparar el cálculo $6200 \div 3$ con el cálculo 12 '400 $\div 6$, que sí da el mismo resultado. Solamente la mitad de los veinte alumnos resolvió de manera correcta este problema; quienes eligieron la respuesta incorrecta justificaron recurriendo a relaciones erróneas, como lo muestra el siguiente ejemplo de Juan: "Parece que fuera lo mismo, pero no, porque tendrían que ser opuestos para ser igual."

También la tercera variante (se agregan/quitan 1000 al dividendo mientras que al divisor se le quita/agrega 1 , p.ej. comparar $24^{\prime} 800 \div 6$ con $23^{\prime} 800 \div 7$ ) dio lugar a confusión; varios alumnos comentaron que estos problemas daban lo mismo, "porque le quitas 1 (que de hecho son 1000) al dividendo y le sumas 1 al divisor". Es interesante que, por un lado, los alumnos consideren que con esa modificación se mantiene el resultado, como si se tratara de una "compensación"; por el otro lado, llama la atención que este razonamiento haya aparecido en diferentes momentos de la secuencia.

La primera vez que se identificó esa "compensación" fue durante un juego de equipos que se hizo en la octava clase. Este juego se llevó a cabo primero con una mitad del grupo, y luego con la otra mitad. Para asegurarse de ganar, al interior de su propio equipo los alumnos tenían que dar a conocer sus estrategias sobre cómo comparar cálculos, tanto de multiplicaciones como de divisiones. Se formaron equipos de 3 o 4 alumnos y se les dio la siguiente consigna:

1. Entre ustedes recuerden y explíquense las diferentes relaciones entre las multiplicaciones y divisiones que hemos encontrado en las sesiones anteriores.

2. Va a pasar un alumno de cada equipo para resolver un problema de comparación entre cálculos. En ese momento su equipo no puede ayudarlo. Por eso es importante que revisen antes las estrategias.

3. Si la respuesta es correcta, el equipo gana un punto. Si la respuesta no es correcta, otro equipo puede ganar el punto si resuelve bien el problema.

4. El equipo que al final tenga más puntos gana el juego. 
Se identificaron ciertas dificultades al resolver este tipo de problemas en las dos partes en que dividimos el grupo para el juego, como se muestra en las siguientes interacciones:

Interacción en la mitad A del grupo:

A Gisela le toca comparar el cálculo 47'120 $\div 13$ con el cálculo 48'120 $\div 12$. Lo pone en "igual a" (el resultado correcto sería "menos que") y explica su decisión:

Gisela: $\quad$ Porque aquí es uno menos (de 48'120 a 47'120) y aquí es uno más (de 12 a 13). (Se ríe, parece insegura.) No sé...

M: $\quad$ Ok, entonces tú dices aquí es uno menos y aquí es uno más, entonces da lo mismo.

Gisela: $\quad$ Creo.

En lo que expresa Gisela, "porque aquí es uno menos y aquí es uno más" se manifiesta la idea de compensación; sin embargo, Gisela exterioriza cierta inseguridad. La docente devuelve el problema al grupo, lo que se puede observar a continuación:

M: Ok. ¿Qué dicen los demás? ¿Quién está de acuerdo con Gisela? ¿Quién dice sí, Gisela tiene razón? Uno, dos, tres... ¿Quién dice Gisela no tiene razón? Uno, dos, tres, cuatro... ¿Quién no está seguro?

Mateo: $\quad$ Yo. (Los demás se ríen.)

M: $\quad$ Ok. ¿Quién quiere explicar? (Melisa levanta la mano.) Melisa.

Melisa (Se queda sentada, piensa.) Aja, aja, aja (asiente con la cabeza.)

M: ¿¿Qué quiere decir esto, Melisa?

Melisa: $\quad$ Que... (Se levanta.) Pienso que Gisela tiene razón, no soy muy inteligente para las matemáticas, pero si este (señala el dividendo del cálculo $49^{\prime} 120 \div 12$ ) y este es más grande y así (señala el pizarrón con las multiplicaciones, pero no está claro a qué se refiere.), entonces uno está más grande y el otro está más pequeño... y pues creo que los dos dan lo mismo.

9 Las transcripciones son traducciones propias del alemán al español. Las expresiones originalmente dichas por los alumnos en español se señalan con letra cursiva. 
Melisa apoya lo expresado por Gisela; sin embargo, no es clara en su explicación: no dice qué tanto más pequeños o más grandes son los números implicados. Después de lo dicho por Melisa quieren participar Lucas y Jorge:

\author{
M: $\quad$ Ok. Lucas, y después Jorge. \\ Lucas: $\quad$ (Se levanta.) Está mal porque... \\ Melisa: $\quad$ Ay (con tono triste). \\ Lucas: $\quad$ Tienes menos dulces (dibuja un dulce en el pizarrón, los demás se ríen) y se los \\ tienes que dar a más niños (dibuja un nin̄o.) \\ Melisa: $\quad$ Ah sí, cierto. (Se levanta y empieza a borrar el dibujo de Lucas.)
}

Lucas expresa su desacuerdo con lo dicho por Gisela y Melisa y argumenta apoyándose en un contexto de reparto para justificar su opinión. Aparentemente la explicación de Lucas le parece lógica a Melisa. Enseguida Jorge apoya a Lucas y da una explicación sin recurrir a ese contexto:

Jorge: $\quad$ Sí, es lo mismo (se levanta.) Es lo mismo que Lucas dijo (termina de borrar el dibujo de Lucas.) Vamos... Para que los dos estén iguales este (el divisor) tiene que estar dividido entre dos o multiplicado por dos (anota debajo de los divisores 11 y $12: \div 2 ; \times 2$ ) y este (el dividendo) igual. (Se refiere a que el dividendo debería de estar dividido entre 2 o multiplicado por 2, igual que el divisor)

Heidi: $\quad O$ entre cuatro, ¿no?

Jorge: $\quad$ Mmm, tienen que... los dos tienen que este... o sea estos dos deben de estar multiplicados o divididos, los dos lo mismo. Pero esto (se acerca al lado del pizarrón con las multiplicaciones) es al revés. Este (uno de los factores) puede estar multiplicado, pero este (el otro factor) tiene que estar dividido. Y tiene que estar siempre dividido o multiplicado por el mismo número. Entonces por eso esto va aquí (mueve el cálculo 47'120 $\div 13$ a la columna "menos".)

La explicación de Jorge, además de no usar ningún otro contexto hipotético, es más amplia que la de Lucas ya que también menciona que son diferentes las relaciones en las multiplicaciones que en las divisiones.

Sin embargo, como se muestra enseguida, a pesar de que Melisa había aceptado la explicación de Lucas, parece volver a entrar en duda con la explicación de Jorge: 
Melisa: $\quad$ Pero tienes más dulces y (en la grabación no se entiende qué más dice. Otros alumnos hacen comentarios que no se entienden.)

Jorge parece darse cuenta que Melisa necesita apoyarse en un contexto para entender el problema, por lo que recurre a la explicación de Lucas:

Jorge: $\quad$ (Parece desesperarse.) Aquí tienes menos dulces y más niños (en 47'120 13) y aquí tienes más dulces y menos niños (en $48^{\prime} 120 \div 12$ ).

Sin embargo, a pesar de las explicaciones recibidas se mantuvo cierta confusión e incertidumbre entre algunos alumnos, como lo manifiesta Heidi:

Heidi: $\quad$ Estoy de acuerdo que este está mal aquí (Se refiere a que el cálculo 47'120 está mal en la columna "igual a") pero es que... es que... estás sumando uno y estás quitando uno, ientonces es muy confuso! Entonces lo hice al azar. Pero, estoy de acuerdo con Jorge, es menos.

Pudimos observar la misma confusión en la otra mitad del grupo, como lo muestran las siguientes interacciones.

Interacción en la mitad B del grupo:

A Alicia le toca comparar el cálculo 47'120 $\div 13$ con el cálculo $48^{\prime} 120 \div 12$. Lo pone en "igual a" (el resultado correcto es "menos que") y explica su decisión:

Alicia: $\quad$ Es igual porque este factor (se refiere al dividendo 47'120) es más pequeño que este (48'120) pero este factor (se refiere al divisor 13) es más grande

Juan: $\quad$ iEs un divisor no un factor!

Alicia: $\quad$ iAh, ah, sí!

M: $\quad$ Explícalo otra vez en español, Alicia, para que todos entiendan lo que dijiste.

Alicia: $\quad$ Este divisor (47'120) es más chiquito que este (48'120) pero este (señala el divisor 13) está más grande. Entonces yo... bueno, ¿ya entendieron?

As:

Sí.

Aunque confunde los términos matemáticos "divisor" y "factor", Alicia expresa la misma idea de compensación que Melisa dio en el grupo anterior. Su 
explicación no es clara y queda inconclusa; sin embargo, sus compañeros expresan haberla comprendido. Laura complementa la explicación de Alicia, apoyando la idea de la compensación, mientras que algunos otros alumnos manifiestan su desacuerdo:

Laura: $\quad$ Como si pusieras del trece uno a siete para que queda... para que (no se entiende en la grabación todo lo que dice, tal vez se refiere a que el divisor 13 es uno más grande que el divisor original, y que el divisor 47, sin considerar los ceros, es uno menos que el divisor 48.)

Alicia: iAja!

Adrián: No, iestá mal!

M: $\quad$ Pregunta: ¿Quién está de acuerdo con Alicia? Levanten la mano. (Levantan la mano José y Pablo.)

Alicia: ¿¿Ya? ¿Sólo tres están de acuerdo conmigo?

M: ¿Quién no está de acuerdo con Alicia? (Levantan la mano Adrián y Pablo.)

Emma: $\quad$ Yo estoy indecisa.

La docente sostiene la incertidumbre por medio de intervenciones diversas de devolución. En este caso solicita a los alumnos que todos se involucren en la resolución del problema por lo que les pide su opinión sobre lo expresado por Alicia. Adrián quiere explicar su desacuerdo.

Adrián: ¿Puedo explicar?

M: $\quad$ Vas Adrián. Puedes hacerlo en español.

Adrián: (Se levanta y va al pizarrón, Alicia sigue enfrente del pizarrón.) Está mal, porque si tú (le habla directamente a Alicia) tienes trece niños, pero tienes cuarenta y siete lápices...

Emma: iAh, está mal!

Adrián: ...tienes menos lápices y más niños entonces, entonces cada niño va a tener menos lápices.

Juan: $\quad$ iAh sí, cierto! (Adrián se sienta, Alicia se queda viendo el pizarrón.)

M: ¿̇Sí Alicia?

Alicia: $\quad$ Sí... pero tú nos dijiste que era... ¿Qué? (Parece confundida. Cambia el cálculo a la columna "menos" y se sienta.) 
Como lo hizo Lucas en el grupo anterior, Adrián se apoya en un contexto de reparto (lápices entre niños) para justificar su respuesta. Alicia, aunque expresa su acuerdo con la explicación de Adrián, parece confundida. La maestra sostiene la incertidumbre y la devolución frente a la convicción de que podría haber otros alumnos que no estuvieran de todo convencidos de la respuesta.

M: $\quad$ Ok, pero creo que José y Blanca todavía no están seguros. Blanca.

Blanca: $\quad$ Sí estoy segura, pero si fuera igual, tendría que ser por dos y por dos. (Se refiere a que si el cociente se mantuviera igual, el dividendo y el divisor se deberían de haber multiplicado por dos.)

M: $\quad$ Ok, ahí sería lo mismo. José.

José: (Se levanta y va al pizarrón. Regresa el cálculo a la columna "igual a".) Porque este es mil menos (se refiere a 47'120 en comparación con 48'120), pero este (se refiere a trece en comparación con 12) es uno más...

M: $\quad$ Entonces, da lo mismo, ¿dices?

Estamos otra vez indecisos, José dice: Aquí son mil menos (lo anota en el pizarrón poniendo una flecha que va de 48'120 a 47'120 y con la anotación 1000 en la flecha) y aquí es uno más, entonces da lo mismo. (Matías levanta la mano.) Matías, ¿qué opinas?

Matías: Está bien.

M: $\quad$ ¿Que da lo mismo?

Matías: Sí.

M: ¿Por qué?

Matías: $\quad$ Porque... espera...sí... porque cuarenta y ocho es cuarenta y ocho, pero baja a cuarenta y siete, pero como que se compensa con el trece que está ahí, porque el otro es doce. Como que se compensa...

A pesar de la explicación de Adrián, José regresa a la idea de la compensación, idea que la docente devuelve al grupo y que Matías apoya. Enseguida Emma expresa su desacuerdo con José y con Matías.
M: iEmma?
Emma: Es menos.
M: ¿Por qué?
Emma: Porque, porque... 
M: $\quad$ Puedes decirlo en español.

Emma: (No contesta. Blanca levanta la mano.)

M: Blanca.

Blanca: $\quad$ Es menos porque a fuerza tiene que ser por dos y por dos, no puede ser más y menos.

Aunque la respuesta de Blanca no es completamente correcta (no tiene que ser "a fuerza" por dos, puede ser multiplicado/dividido por cualquier número), Blanca parece haberse dado cuenta de que no es posible que el resultado de dos divisiones sea igual si los dividendos y los divisores se distinguen por un número que se suma o se resta.

$\begin{array}{ll}\text { Emma: } & \text { Ya sé! (se levanta y va al pizarrón.) } \\ \text { Emma: } & \begin{array}{l}\text { Porque mira, este es más grande que este (se refiere al divisor } 13 \text { en } \\ \text { ción con 12) y este (el dividendo) es menos, entonces es lógico que tien } \\ \text { menos. }\end{array} \\ \text { M: } & \text { ¿Por qué es lógico? } \\ \text { Emma: } & \text { iPorque así es! } \\ \text { M: } & \text { ¿Por qué? } \\ \text { Emma: } & \text { iPorque sí! (Cambia el cálculo a la columna "menos que" y se sienta.) } \\ \text { Matías: } & \text { ¿Por qué dices que sí, si ni siquiera sabes por qué es? }\end{array}$

Sigue una discusión acalorada en la cual Emma insiste que "Es lógico que no puede dar lo mismo", mientras que Matías vuelve a recurrir a la idea de la compensación.

M: $\quad$ Alicia.

Alicia: $\quad$ No entiendo a Emma, porque dice es que este es más grande que este, pero este es más chiquito, entonces es muy obvio que tenga que ser menos. (Dice algo más que no se entiende bien en la grabación. Mientras que Alicia habla, se levanta Matías y regresa el cálculo otra vez a la columna "igual a".)

Matías: $\quad$ Si quitas el siete (de 47'120) y pones el ocho, sería lo mismo. Y si aquí (divisor) si le restas uno te da doce, entonces si aquí le restas uno, te da cuarenta y siete y si aquí le sumas uno te da trece. Es como que se compensa el número que... 


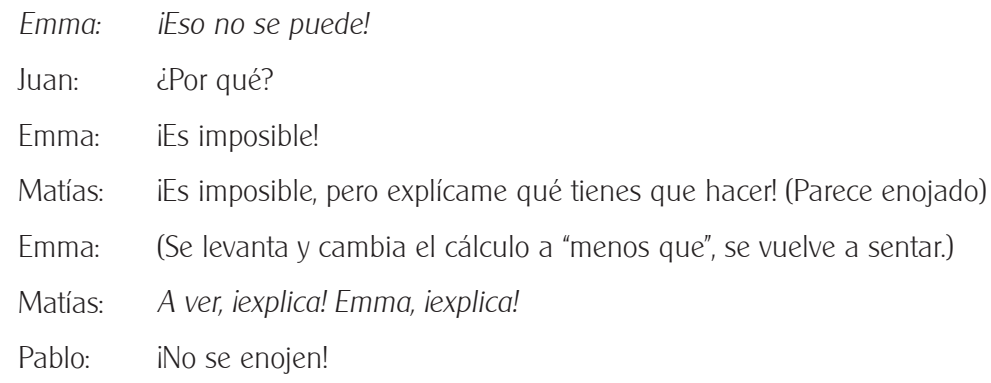

Para calmar la situación la docente realiza una intervención en la cual solicita a Adrián que repita su explicación. Esta intervención se apoya en el supuesto de que, para algunos alumnos, escuchar la explicación contextualizada por parte de su compañero les permitirá entender las relaciones en juego:

M: $\quad$ Escuchen bien la explicación de Adrián.

Adrián: Es menos, porque hazte cuenta que tienes cuarenta y siete lápices, bueno cuarenta y siete mil ciento veinte, pero tienes trece niños, o sea más niños... y menos lápices. Obviamente...

Matías: iAh claro!

Adrián: $\quad$...si tienes más niños y menos lápices cada uno recibe menos.

As: $\quad$ iSí!

M: ¿Convencimos a todos? ¿josé?

José: $\quad$ Asiente con la cabeza.

M: ¿ ¿Seguros? ¿Alicia? ¿Juan?

As: $\quad$ iŚ!

En los extractos anteriores podemos identificar que a pesar de las argumentaciones apoyadas en un contexto de reparto que dieron algunos compañeros, hay otros que volvieron a recurrir a la justificación errónea de la compensación por "quitarle uno al dividendo y sumarle uno al divisor."

En la novena clase se retomó el mismo ejemplo para la elaboración grupal de un cartel; el propósito era que el cartel resumiera las relaciones entre multiplicaciones y entre divisiones: 
Alicia: $\quad$ Si el dividendo es más pequeño y el divisor más grande, no da el mismo resultado.

M: $\quad$ Entonces, por ejemplo, como aquí (señala el cálculo 47'120 $\quad 13$.)

Alicia: iAja!

M: $\quad$ Alicia dice: Si el dividendo es más pequeño y el divisor más grande, no da el mismo resultado. En este cálculo, en los dos grupos de ayer hubo discusiones.

As: $\quad$ iSí!

M: $\quad$ ¿Por qué hubo discusiones? ¿Se acuerdan? Porque algunos lo pusieron en medio (se refiere a la columna de "igual a", pega el cálculo ahí.) ¿Se acuerdan por qué algunos, al principio dijeron que daba lo mismo? Carla.

Carla: $\quad$ (No se entiende bien lo que dice en la grabación.)

M: $\quad$ Exacto, algunos ayer dijeron, aquí dice más uno y acá menos uno (pone flechas con $+1 /-1$ de los números originales a los números del cálculo que se compara) y da lo mismo. Y ahora dice Alicia: No, yo aprendí que no da lo mismo. ¿Podemos, en lugar de solamente anotar que no da lo mismo, decir si da menos o más? José.

José: $\quad$ Da menos.

M: ¿¿Por qué da menos?

José: $\quad$ Porque el dividendo es menos y el divisor es más. Si tienes más niños y menos lápices cada uno de los niños recibe menos.

M: $\quad$ Bien, entonces José explica con el mismo ejemplo. (Anota el ejemplo y la explicación en el cartel.) José dice: Aquí tenemos menos lápices y más niños. Por eso da... ¿Da qué Pablo? ¿Menos lápices por niños o más lápices por niño? (Le solicita a Pablo que conteste porque lo ve distraído.)

Pablo: $\quad$ Mhhh, menos.

M: $\quad$ Menos. Bien, ¿algo más que podríamos anotar acerca de la división? Melisa.

Melisa: $\quad Y$ si tienes lo contrario, si tienes más en el primero y menos en el segundo, tienes más porque tienes más cosas que dar y menos a quienes repartir.

Lo expresado por los alumnos en este momento de la clase hizo pensar a la maestra que se habían convencido de que no existe una "compensación" en este caso. Sin embargo, en la evaluación que se hizo posteriormente en la clase 10, ante la comparación de los cálculos 17 '800 $\div 7$ y $18^{\prime} 800 \div 6$, pudimos identificar que al menos dos alumnos seguían con la misma confusión, como se 
muestra en lo escrito por Ana: "Es igual porque al primer numero ${ }^{10}$ le quitaste uno y al que estas dividiendo le sumaste uno" y por Pedro: "Es igual porque como un factor principal es menor y el otro es mayor hace que sea algo igual."

Catorce de veinte alumnos contestaron de manera correcta el problema de comparar el cálculo $17^{\prime} 800 \div 7$ con el cálculo $18^{\prime} 800 \div 6$, tres contestaron que los dos cálculos daban lo mismo, dos contestaron que el segundo daba más que el primero y un alumno no contestó el problema. De los tres alumnos que contestaron erróneamente, Ana y Pedro justificaron con una compensación, mientras que Laura pareció confundirse entre las relaciones que se pueden establecer entre dos multiplicaciones y las que se establecen entre dos divisiones. Esta última alumna escribió: "si el primer número es entre y el segundo es por o viceversa es igual", lo cual sí puede ser correcto en la comparación de dos multiplicaciones (por ejemplo $4 \times 2400=2 \times 4800$ ) pero no en una división $(2400 \div 4<4800 \div 2)$. Además, en su explicación sobre por qué eligió "igual a 18 '800 $\div$ 6" mencionó una relación multiplicativa que no se encuentra en el problema, como se muestra en su respuesta escrita: "Yo digo que da igual porque Sandra (la maestra) nos enseño que si el primer numero es entre y el segundo por o visebersa es igual."

\section{CONCLUSIONES}

Para la resolución de problemas que implican una comparación de divisiones, de manera semejante a lo que hicieron en las multiplicaciones, los alumnos recurrieron a analizar las diferencias entre el dividendo y el divisor de un cálculo, con el dividendo y el divisor del otro cálculo, para después establecer relaciones entre los dos cálculos. Les resultó más fácil establecer relaciones en problemas donde solamente era diferente el dividendo o el divisor, aunque en ocasiones hubo confusión respecto a qué relaciones eran válidas para las multiplicaciones y cuáles para las divisiones. Causaron más dificultad aquellos problemas en los que eran diferentes el dividendo y el divisor. Observamos que si bien los alumnos identificaron qué relación había entre el dividendo y el divisor de un cálculo y el otro (por ejemplo: "se dividió el dividendo entre dos y se multiplicó el divisor por dos"), se confundieron con los efectos que estas diferencias tenían sobre el

10 En todas las transcripciones de respuestas escritas por los alumnos se respetaron la redacción y la ortografía originales. 
resultado. Podemos resaltar que para facilitar el trabajo con dichas relaciones la maestra, aunque no estuviera previsto en el análisis a priori, promovió que los alumnos se apoyaran en contextos hipotéticos que les ayudaran a interpretar las relaciones involucradas. La mayoría de los alumnos se apoyó en este recurso para resolver problemas que exigen una comparación de divisiones en varios momentos de la secuencia, a veces de manera espontánea y otras después de una invitación de la docente.

Pudimos observar también que durante la secuencia hubo alumnos que seguían con dificultades con las relaciones enseñadas, a pesar de haberlas institucionalizado a lo largo de varias sesiones, y a pesar de haber analizado que las relaciones entre multiplicaciones y entre divisiones no son iguales. Por un lado, esto nos indica que establecer estas relaciones es efectivamente complejo, y que se necesita un trabajo continuo que permita que los conocimientos sobre ese aspecto se puedan consolidar. Por el otro lado, identificamos que los problemas propuestos son sumamente fructíferos para trabajar las relaciones que se pueden establecer entre dos multiplicaciones y entre dos divisiones.

Varios autores plantean que este tipo de problemas puede aportar al desarrollo del pensamiento algebraico, sobre todo en cuanto a temas de equivalencia entre dos expresiones y a la posibilidad de tratar relaciones entre expresiones sin exigir un resultado numérico. Las aportaciones de este tipo de problemas son aún más relevantes si se consideran las siguientes limitaciones en las prácticas escolares clásicas de la escuela primaria, las cuales ya han sido señaladas por diversos trabajos de investigación: la ausencia de situaciones que exijan comparar cálculos y la presentación del símbolo igual solamente como antecesor del resultado de un cálculo que se escribirá a la derecha de este símbolo. En cambio, los problemas que exigen la comparación de cálculos abonan a una ampliación de los sentidos del símbolo igual (Broitman, Castillo y Bernasconi Echeverría, 2019), enriqueciendo así los conocimientos aritméticos y sin limitar el desarrollo de un pensamiento algebraico.

Como hemos mencionado en diferentes momentos de este artículo, lograr que los alumnos elaboren conocimientos que vayan más allá de los cálculos exactos y de los algoritmos es un propósito importante en la enseñanza. Para finalizar expresamos nuestra intención de que este estudio colabore con la enseñanza del cálculo estimativo en la escuela primaria y aporte al necesario desarrollo de nuevas investigaciones didácticas. 


\section{REFERENCIAS}

Artigue, M. (1998). Ingeniería didáctica. En M. Artigue, R. Douady, L. Moreno, y P. Gómez, Ingeniería didáctica en educación matemática (pp. 33-60). Grupo Editorial Iberoamérica S.A.

Broitman, C. (2011). Estrategias de cálculo con números naturales: Segundo ciclo. Santillana.

Broitman, C. (2012). Adultos que inician la escolaridad: sus conocimientos aritméticos y la relación que establecen con el saber y con las matemáticas. (Tesis doctoral no publicada). Facultad de Humanidades y Ciencias de la Educación. Universidad Nacional de la Plata.

Broitman, C., Castillo, C., y Bernasconi Echeverría, A. (2019). Hacia la ampliación de sentidos del símbolo igual en los primeros grados de la escuela primaria. YUPANA Revista de Educación Matemática de la Universidad Nacional del Litoral 11, 8/37.

Broitman, C., Itzcovich, H., Novembre, A., Escobar, M., Grimaldi, V., Ponce, H., y Sancha, I. (2015). Los matemáticos de $4^{\circ}$. Santillana.

Brousseau, G. (2007). Iniciación al estudio de la teoría de las situaciones didácticas. Libros del Zorzal.

Lerner, D. (2005). ¿Tener éxito o comprender? Una tensión constante en la enseñanza y el aprendizaje del sistema de numeración. En M. Alvarado, y B. M. Brizuela, Haciendo Números (pp. 147-197). Paidós.

Mason, J., Graham, A., Pimm, D., y Gowar, N. (1999). Rutas hacia el álgebra. Universidad Pedagócica y Tecnológica de Colombia.

Ministerio de Educación, Ciencia y Tecnología. (2005). Núcleos de Aprendizajes Prioritarios. Segundo Ciclo EGB/Nivel Primario. Ministerio de Educación, Ciencia y Tecnología.

Saiz, I., y Parra, C. (2013). Hacer Matemática 5. Estrada.

Secretaría de Educación Pública. (2011). Programas de estudio 2011: Guía para el Maestro. Educación Básica Primaria. Quinto Año. SEP.

Secretaría de Educación Pública. (2016). Desafíos Matemáticos. Libro para el Maestro. Quinto Grado. SEP.

Secretaría de Educación, G. (2006). Cálculo mental con números naturales: apuntes para la enseñanza. Coordinado por Susana Wolman. Gobierno de la Ciudad de Buenos Aires.

Segovia, I., Castro, E., Castro, E., y Rico, L. (1989). Estimación en cálculo y medida. Síntesis. 
Stauffer, S. (2018). Cálculo estimativo en quinto grado de primaria. Implementación de una secuencia didáctica. (Tesis de maestría no publicada). Universidad Autónoma de Querétaro.

SANDRA STAUFFER

Dirección: Universidad Autónoma de Querétaro

Cerro de las Campanas s/n, Colonia las Campanas 76010 Santiago de Querétaro, QRO

Teléfono: 4421393925 